\title{
TIROIDECTOMÍA TRANS-ORAL ENDOSCOPICA CON ABORDAJE VESTIBULAR (TOETVA) VERSUS TIROIDECTOMÍA ENDOSCÓPICA: COMPLICACIONES POSTQUIRURGICAS.
}

\author{
Frías Guevara María Isabel ${ }^{1 *}$, Arias Terán Elva Beatriz ${ }^{1}$
}

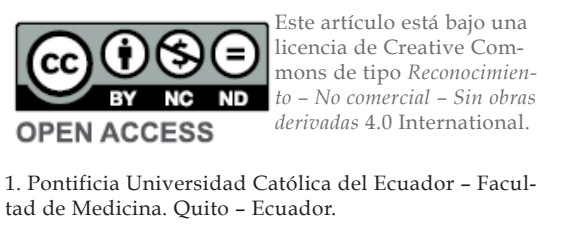

Frías María Isabel

orcid.org/0000-0003-2933-8200

Arias Elva Beatriz

orcid.org/0000-0003-3610-2503

*Corresponding author: Frías María Isabe

E-mail: mariaisabelfriasg@gmail.com

\section{Article history}

Received: 19 - May - 2020

Accepted: 30 - Sep - 2020

Publish: 01 - Jun - 2021

STROBE 2008 Check List statement: Th

authors have real the STROBE 2008 Check List and the manuscript was prepared and revised according to the STROBE 2008 Checklist.

Conflict of interest: All authors declared that there are no conflicts of interest.

Financial disclosure: The authors have no financial relationships relevant to this article to disclose.

Authors' contribution: All the authors reviewed and approved the final manuscript.

\section{Resumen \\ Introducción}

La excéresis de la glándula tiroides es realizada desde el siglo XIX la cual fue empleada por primera vez por Theodor Kocher, esta técnica además de presentar ciertas complicaciones postquirúrgicas, deja una cicatriz estéticamente incómoda para los pacientes, sin embargo hace unos años atrás se han venido realizando técnicas bajo abordajes laparoscópicos los cuales dejan menos cicatrices, bajo este mismo concepto, en el año 2016 se presenta la técnica TOETVA (Tiroidectomía trans-oral endoscópica con abordaje vestibular) con la cual se ha evidenciado menores complicaciones postquirúrgicas.

\section{Objetivo}

Identificar complicaciones postquirúrgicas, tiempo quirúrgico y de hospitalización entre las técnicas TOETVA versus endoscópica.

\section{Materiales y Métodos}

Se realizó un estudio retrospectivo de dos intervenciones quirúrgicas, en el cual se incluyeron pacientes intervenidos mediante Tiroidectomía Trans-oral Endoscópica con abordaje vestibular y pacientes intervenidos mediante Tiroidectomía Endoscópica Cervical en el Hospital General IESS Ibarra en el período comprendido entre Enero 2017 - Marzo 2019.

Treinta y tres pacientes cumplieron con los criterios de inclusión, 19 pacientes fueron intervenidos mediante Tiroidectomía Trans-oral Endoscópica con abordaje vestibular TOETVA, y 14 con Tiroidectomía endoscópica cervical.

El análisis estadístico descriptivo e inferencial se realizó mediante el software SPSS. Versión 19.

\section{Resultados}

Se estudiaron un total de 33 pacientes, de los cuales el 100\% de la población estudiada correspondieron al sexo femenino, con una edad promedio de 45,3 años, el 42,42\% residen en lbarra, mientras que el 15,5\% correspondía a pacientes provenientes de Otavalo, estas dos poblaciones fueron las más representativas.

En cuanto a complicaciones el $68,4 \%$ de los pacientes intervenidos por Tiroidectomía Trans-oral Endoscópica con abordaje vestibular (TOETVA) no presentaron complicaciones, por su parte el $71,42 \%$ de pacientes intervenidos por Tiroidectomía endoscópica cervical no presentó complicaciones.

También se estudió el tiempo quirúrgico en el que se encontró que la Tiroidectomía Endoscópica Trans-oral con abordaje vestibular (TOETVA), tuvo una media de 129.84 minutos, mientras que la Tiroidectomía endoscópica Cervical tuvo una media de 131.07 minutos.

La estancia hospitalaria fue menor para la tiroidectomía endoscópica cervical debido a las complicaciones presentadas con TOETVA con una media de 6,32 y 4,14 días respectivamente.

\section{Conclusiones}

La Tiroidectomía Trans-oral Endoscópica con abordaje vestibular (TOETVA) es una técnica que se encuentra en proceso de implementación y aprendizaje en Ecuador y que a raíz de la presente investigación se pudo concluir que no existen diferencias significativas respecto de las complicaciones postquirúrgicas entre las técnicas estudiadas.
Forma de citar este artículo: Frías MI, Arias EB. A. TIROIDECTOMÍA TRANSORAL ENDOSCOPICA CON ABORDAJE VESTIBULAR (TOETVA) VERSUS TIROIDECTOMÍA ENDOSCÓPICA: COMPLICACIONES POSTQUIRURGICAS. Rev Med Vozandes. 2021; 32 (1): 23-31 
Keywords: TOETVA Thyroidectomy, Endoscopic Thyroidectomy, Surgical Complications, Thyroid.

\section{Abstract \\ TRANSORAL ENDOSCOPIC THYROIDECTOMY VESTIBULAR APPROACH (TOETVA) VERSUS ENDOSCOPIC THYROIDECTOMY: POST-SURGICAL COMPLICATIONS.}

\section{Introduction}

thyroid gland surgical excision has been performed since the 19th century, which was first employed by Theodor Kocher, this technique since its inception presented certain post-surgical complications and left an aesthetically uncomfortable scar in patients; however a few years ago new techniques have been carried out under laparoscopic approaches which leave less scarring; under this same concept, in 2016 the TOETVA technique (Endoscopic transoral thyroidectomy with vestibular approach) is presented, with which fewer postoperative complications have been evidenced.

Objective

To identify post-surgical complications, surgical and hospitalization time between TOETVA versus endoscopic techniques.

\section{Materials and Methods}

a retrospective study of two surgical interventions was carried out, which included patient's surgery by Endoscopic Trans-oral Thyroidectomy and patient's surgery by other endoscopic techniques at Hospital General IESS Ibarra in the period between January 2017 - March 2019.

Thirty-three patients met the inclusion criteria, 19 patients were tested for Endoscopic Transoral Thyroidectomy with TOETVA vestibular approach, and 14 with cervical endoscopic thyroidectomy.

Descriptive and inferential statistical analysis was performed using SPSS software. Version 19.

Results

the entire studied population was female, with 45.3 years old in average, $42.42 \%$ resided in Ibarra, while $15.5 \%$ were from Otavalo, these two populations were the most representative.

Regarding to the issue, $68.4 \%$ patient's surgery by TOETVA technique and $71.42 \% \%$ patient's surgery by cervical endoscopic thyroidectomy technique did not present complications. The surgical time in which it was reported that Endoscopic Trans-oral Thyroidectomy with vestibular approach was also studied, TOETVA had a mean of 129.84 minutes, while the cervical endoscopic had a mean of 131.07 minutes. The hospital stay was shorter for cervical endoscopic thyroidectomy due to complications presented with TOETVA with a mean of 6.32 and 4.14 days respectively.

\section{Conclusions}

The prevalence of DM2 among adults over 75 years of age was 14\%, and the presence of chronic complications was related to the longer evolution time of the disease along with higher $\mathrm{HbAlC}$ values. 


\section{Introducción}

Ciertas patologías que afectan a la glándula tiroides son de resolución quirúrgica, para lo cual existen dos métodos principales para el abordaje de esta, los cuales incluyen el abordaje abierto y las variantes de abordajes endoscópicos. A finales del siglo XIX Theodor Kocher realiza por primera vez la extirpación de la glándula tiroides por abordaje abierto, sin embargo esta técnica deja al paciente con una cicatriz estéticamente indeseable, en 1997 Huscher realiza por primera vez la excéresis de la glándula tiroides mediante endoscopía, con la utilización de dos abordajes cervicales y extracervicales, sin embargo aún quedan pequeñas cicatrices visibles, causa mayor tiempo quirúrgico y el riesgo de complicaciones postquirúrgicas aumenta. $(1-3,20,31,33)$

Además de la técnica descrita existen abordajes a nivel axilar, axilo-mamario y retroauricular, los cuales varían en su tiempo quirúrgico pero también dejan cicatrices en el paciente, sin embargo mediante la cirugía natural orifice transluminal endoscopic surgery ${ }^{(25,27)} \mid a$ cual se desarrolla a través de los orificios corporales naturales, que no dejan cicatriz, minimiza el tiempo quirúrgico ${ }^{(20)}$, el daño tisular, disminuye la morbilidad y la recuperación del paciente es en menor tiempo. ${ }^{(28)}$ En el año 2016 Anuwong ${ }^{(1,4)}$ describe una serie de 42 casos para lobectomía tiroidea y 22 casos para tiroidectomía total mediante la tiroidectomía trans-oral endoscópica (TOETVA), posteriormente Witzel et al. realiza la resección endoscópica en un modelo porcino y Benhidjeb et al. realiza la resección tiroidea en modelos cadavéricos, como resultado se ha empleado esta técnica alrededor del mundo para enfermedades tiroideas de resolución quirúrgica. ${ }^{(5-7)}$

En el país se realiza esta técnica por primera vez en el Hospital General IESS Ibarra en el año 2016, exponiendo importantes resultados como la integridad anatómica de nervios laríngeos y glándulas paratiroides, sangrado mínimo, reducción tanto del tiempo quirúrgico como de las complicaciones postquirúrgicas, por lo cual realizan formalmente el reporte de esta cirugía en la cual concluye que es fácilmente reproducible en el país ya que no se requiere de instrumental especial, salvo el equipo laparoscópico convencional. ${ }^{(8,5,33)}$

En el caso de TOETVA no es indicativo de este procedimiento pacientes con tiroidectomía previa, tamaño de glándula tiroides mayor a $10 \mathrm{~cm}$, pacientes sometidos previamente a radiación en cabeza, cuello o mediastino, pacientes con nódulos dominantes superiores a $50 \mathrm{~mm}$, invasión traqueal o esofágica, parálisis de nervio laríngeo recurrente, evidencia de metástasis a distancia. ${ }^{(9-13,15)}$

En el caso de los otros tipos de cirugía por abordaje endoscópico los principales factores que limitan a esta técnica de tiroidectomía mínimamente invasiva incluyen la obesidad del paciente, el tamaño del nódulo y/o la glándula tiroides, y la presencia de tiroiditis. $(8,11,19)$

\section{Pacientes y Métodos}

\section{Diseño del estudio}

Se realizó un Estudio de cohorte retrospectivo, descriptivo, comparativo de pacientes mayores de 18 años, de ambos géneros, atendidos entre Enero del 2017 hasta Marzo 2019, en el servicio de Cirugía del Hospital General IESS Ibarra.

Se excluyeron a todos los pacientes sometidos a tiroidectomías diferentes a las estudiadas.

Toda la información relacionada con los procedimientos quirúrgicos, tales como sangrado y complicaciones postquirúrgicas fueron recolectadas mediante revisión de historias clínicas de los participantes (Sistema AS400).

\section{Consideraciones éticas}

Este estudio sigue los lineamientos para estudios observacionales mediante la declaración STROBE ( Strenghtening the Reporting of Observational Studies in Epidemiology), el mismo contó con la aprobación del comité de bioética de la Facultad de Medicina de la Pontificia Universidad Católica del Ecuador y del Hospital General IESS Ibarra.

\section{Análisis estadístico}

El análisis estadístico lo realizamos con IBM SPSS software package, version 19.0 for Windows (IBM, Armonk, NY, USA). Los datos cuantitativos se describen como media y desviación estándar, mientras que los datos categóricos se presentaron como contajes y porcentajes. Las comparaciones entre variables de interés se realizaron con el test Shapiro-Wilk.

Para las comparaciones entre medias fueron realizadas por el test $t$ de Student o por el test U de Mann-Whitney en las situaciones de asimetría. Los datos categóricos fueron comparados por chicuadrado. Valores de $\mathrm{P}<0,005$ se consideraron estadísticamente significativos.

\section{Resultados}

Se evaluó entre el 17 de marzo de 2017 al 9 de enero de 2019 a 60 pacientes con patología tiroidea plausible de resolución quirúrgica en el Hospital general IESS Ibarra.

De estos, 27 fueron excluidos por pertenecer al grupo de pacientes intervenidos mediante abordajes diferentes a Tiroidectomía Trans-oral Endoscópica y Tiroidectomía endoscópica.

Del grupo de los 33 pacientes que cumplían los criterios de inclusión, se dividió en subgrupos de acuerdo al abordaje endoscópico: 19 pacientes mediante la Técnica TOETVA, y 14 pacientes mediante la Técnica endoscópica Cervical. 
Ilustración 1 Criterios de Inclusión y exclusión

\section{0 pacientes con patología tiroidea de resolución QX}
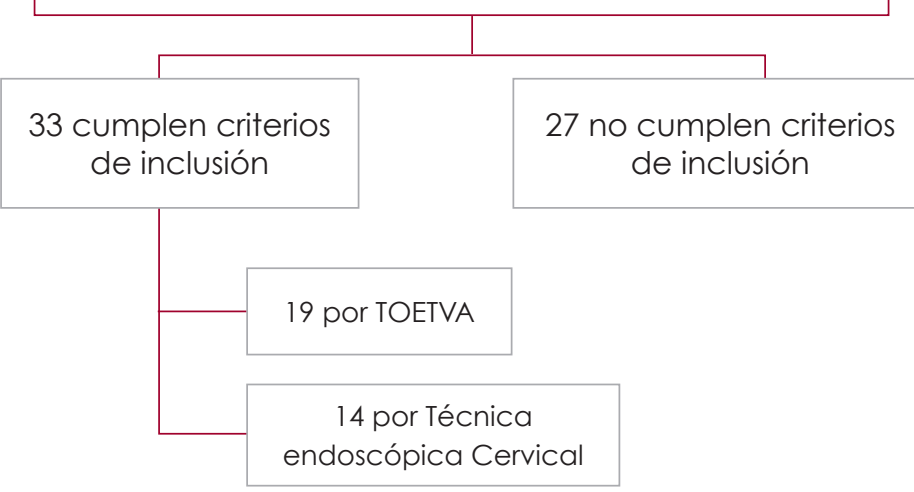

Fuente: Elaborado por los autores
Tabla 1 Datos demográficos y quirúrgicos de acuerdo al abordaje quirúrgico, tomando como punto de cohorte la mediana de la muestra.

Variable $n=33$ TOETA $\begin{aligned} & \text { Tiroidectomía } \\ & \text { Endoscópica }\end{aligned}$

EDAD $n, \%$

$\begin{array}{lcc}28 \text { a } 38 \text { años } n, \% & 6(18,18) & 2(6,1) \\ 39 \text { a } 49 \text { años } n, \% & 6(18,18) & 7(21,21) \\ 50 \text { a } 59 \text { años } n, \% & 5(15,15) & 1(3,0) \\ >\text { 60 años } n, \% & 2(6,1) & 4(12,12)\end{array}$

\section{TIEMPO}

\section{QUIRURGICO}

$\leq 110$ minutos $n, \% \quad 7(21,21) \quad 5(15,15)$

$>110$ minutos $n, \% \quad 12(36,36) \quad 9(27,27)$

$\begin{array}{lll}\text { Total } & 19 & 14\end{array}$

Fuente: Elaborado por los autores

Gráfico No. 1 Diagnósticos para abordaje por TOETVA

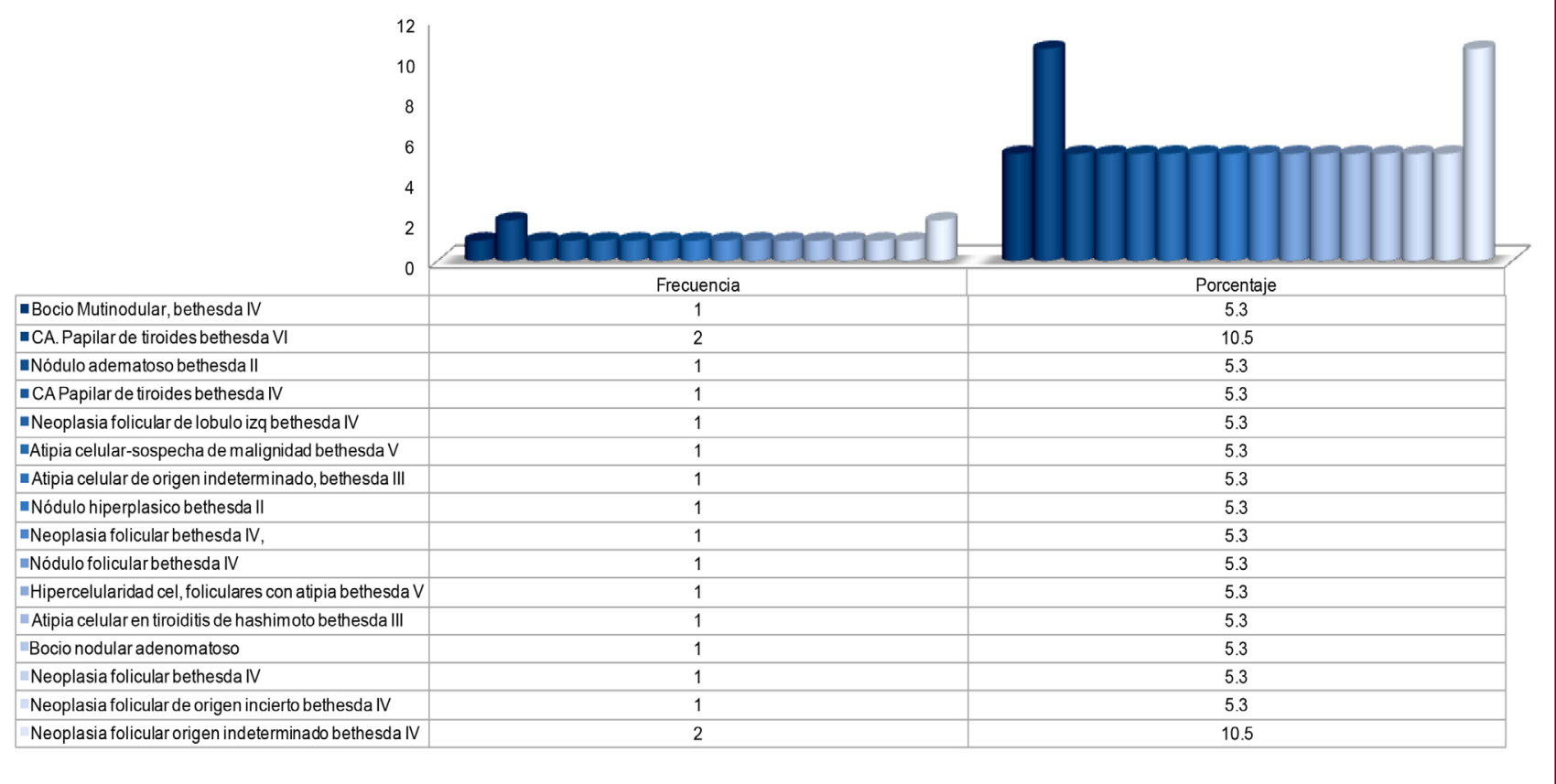


Gráfico No. 2 Diagnósticos para abordaje por Tiroidectomía Endoscópica

- Papilar de tiroides lóbulo izq. bethesda VI metastasico

- CA. Papilar de tiroides bethesda VI

- Nódulo lóbulo izq, células foliculares con atipia bethesda V

øCA. Papilar de tiroides bethesda VI

- Nódulo hiperplásico - células foliculares bethesda II

- Pleomorfismo celular con células colides bethesda $V$

ఐ Nódulo con células hiperplasicas bethesda $\mathrm{V}$

Nódulo hiperplásico bethesda II + nódulo lóbulo der. ca. papilar de tiroides bethesda $V I$

CA.Papilar de tiroides lóbulo izq bethesda VI

CA. Papilar de tiroides bethesda V

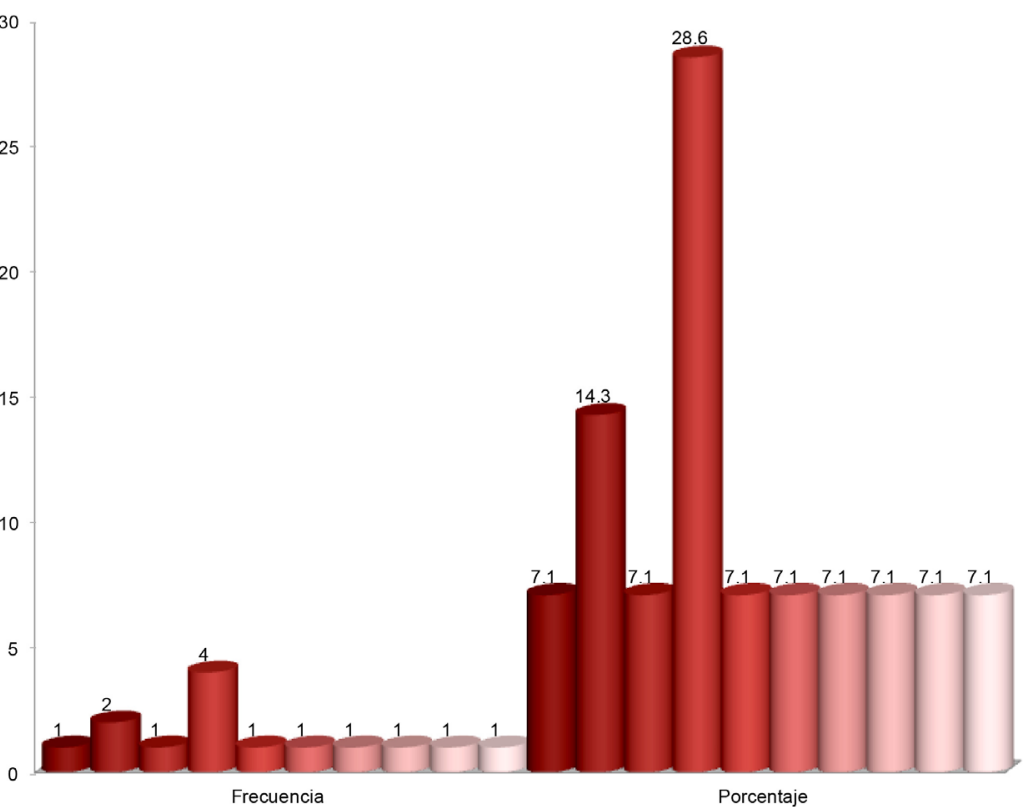

Fuente: Elaborado por los autores

Gráfico No. 3 Pacientes sometidos a cirugía total, cirugía parcial y vaciamiento, Técnica quirúrgica

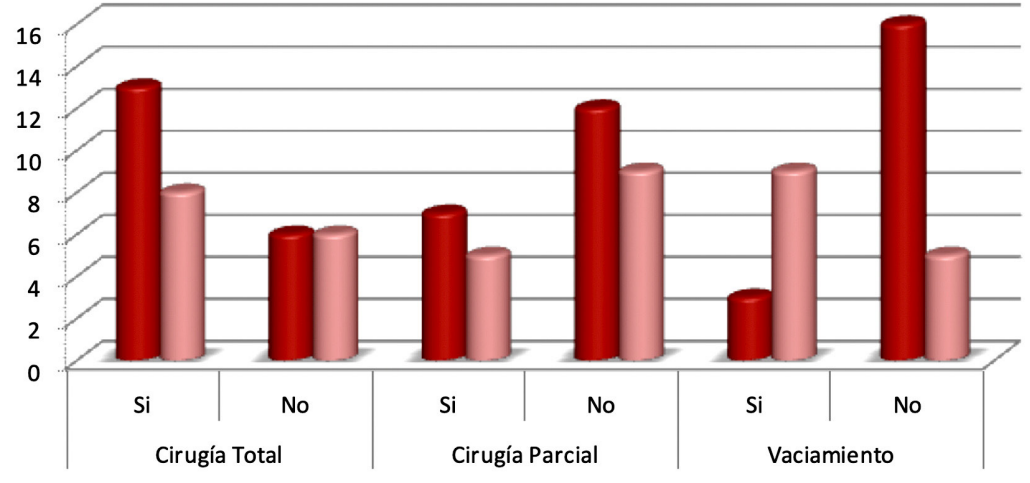

- TOETVA $\square$ Endoscópica

Fuente: Elaborado por los autores 


\begin{tabular}{|c|c|c|c|c|}
\hline \multicolumn{5}{|c|}{$\begin{array}{l}\text { Tabla } 2 \text { Pruebas de Norm } \\
\text { Pruebas de normalidad }\end{array}$} \\
\hline \multirow{2}{*}{$\begin{array}{l}\text { Técnica } \\
\text { Quirúrgica }\end{array}$} & \multirow[t]{2}{*}{ Variable } & \multicolumn{3}{|c|}{ Shapiro-Wilk } \\
\hline & & Estadístico & gl & Sig. \\
\hline \multirow[t]{2}{*}{ TOETVA } & Tiempo Quirúrgico &, 924 & 19 &, 136 \\
\hline & Estancia Hospitalaria &, 574 & 19 &, 000 \\
\hline \multirow{2}{*}{$\begin{array}{l}\text { Endoscó- } \\
\text { pica }\end{array}$} & Tiempo Quirúrgico & ,891 & 14 &, 084 \\
\hline & Estancia Hospitalaria & ,909 & 14 & 150 \\
\hline
\end{tabular}

\begin{tabular}{|c|c|c|c|c|}
\hline \multirow[t]{3}{*}{$\begin{array}{l}\text { Tabla } 3 \\
\text { Técnica } \\
\text { Quirúrgica }\end{array}$} & \multicolumn{2}{|c|}{$\begin{array}{l}\text { Distribución } \\
\text { T-Student }\end{array}$} & \multicolumn{2}{|c|}{$\begin{array}{l}\text { Prueba U de } \\
\text { Mann-Whitney }\end{array}$} \\
\hline & \multicolumn{2}{|c|}{$\begin{array}{l}\text { Tiempo } \\
\text { Quirúrgico }\end{array}$} & \multicolumn{2}{|c|}{$\begin{array}{l}\text { Estancia } \\
\text { Hospitalaria }\end{array}$} \\
\hline & Media & p-valor & Media & p-valor \\
\hline TOETVA & 129,84 & 0,945 & 6,32 & 0,461 \\
\hline Endoscópica & 131,07 & & 4,14 & \\
\hline
\end{tabular}

Gráfico No. 4 Otras complicaciones derivadas de TOETVA

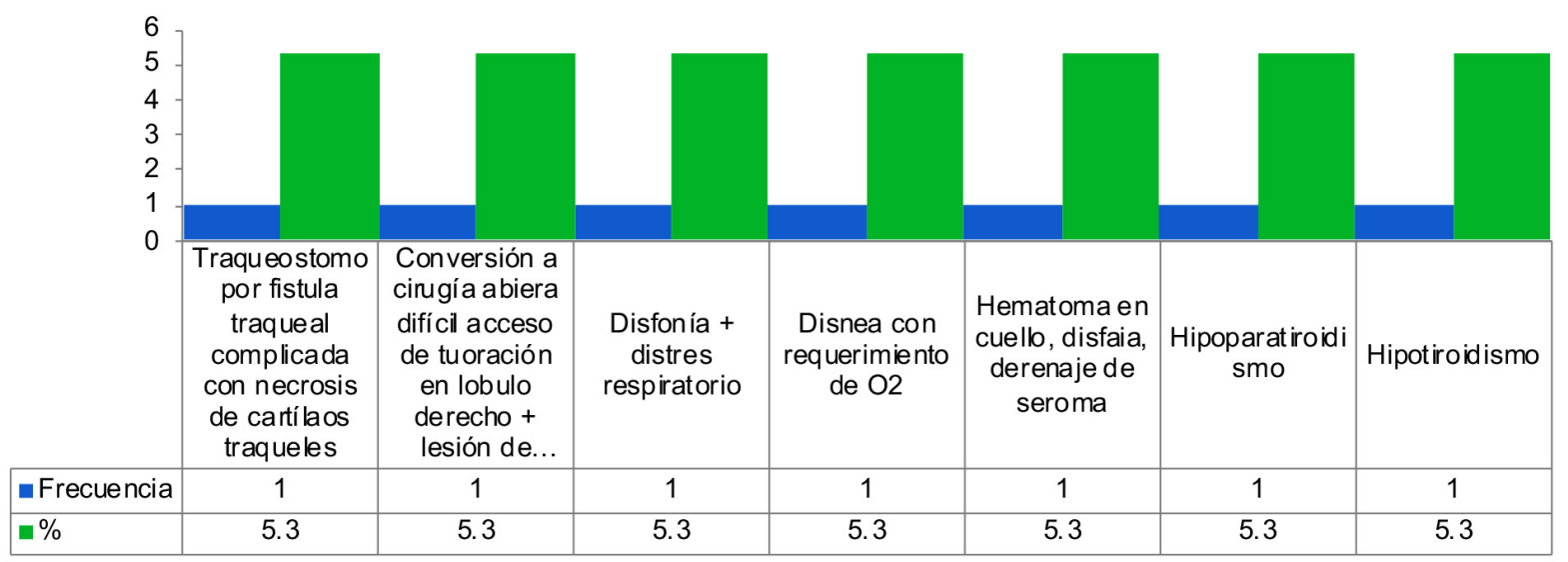

Gráfico No. 5 Otras complicaciones derivadas de la técnica Endoscópica Cervical

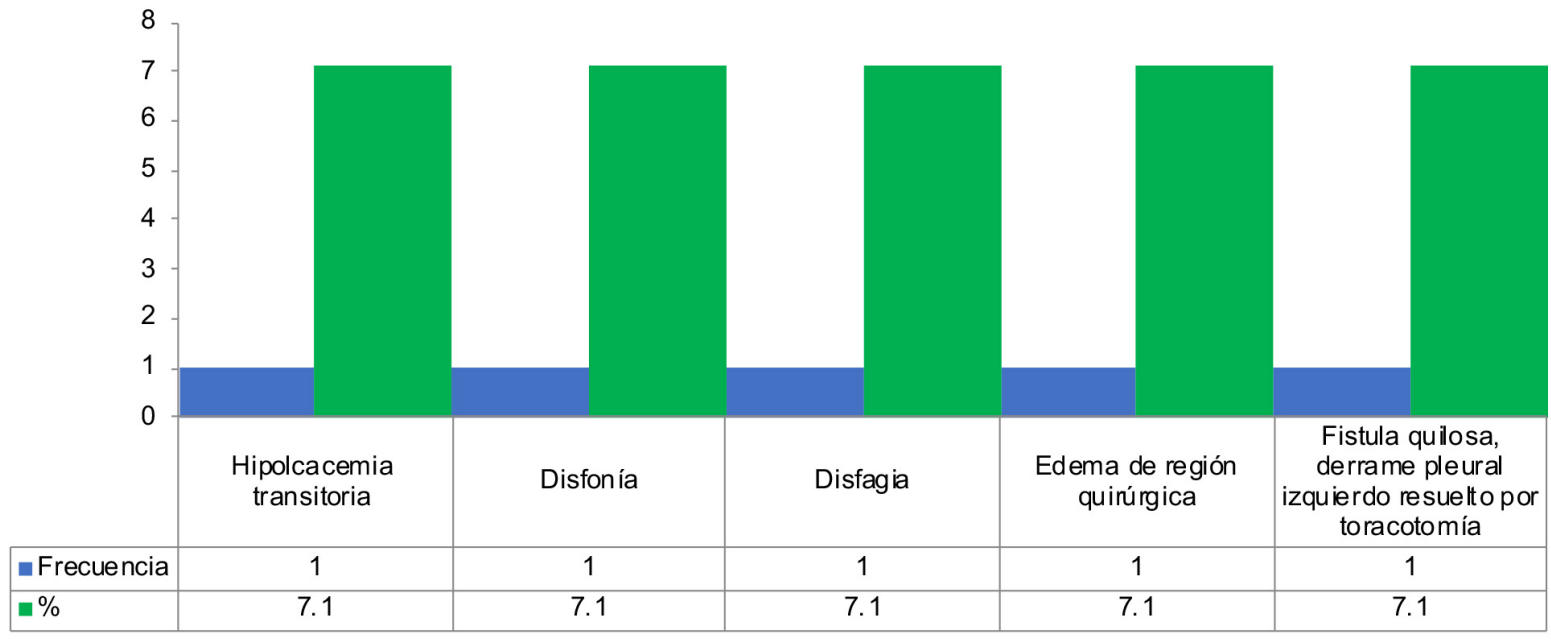


Para el análisis estadístico del tiempo quirúrgico se tomaron datos de estudios realizados previamente a nivel internacional tomando como referencia 110min, además se tomó en cuenta el tiempo y el número de casos requeridos para una curva de aprendizaje promedio para la técnica TOETVA. $(6,16,17)$

Con el propósito de realizar prueba de hipótesis para analizar si existen diferencias significativas entre el tiempo quirúrgico y la estancia hospitalaria entre ambas técnicas se hacen en primer lugar pruebas de normalidad para la verificación de supuestos requeridos por dichas técnicas y así poder seleccionar la más apropiada.

Las pruebas de normalidad aplicadas a la población asociada con la técnica TOETVA fueron consideradas con una desviación estándar de (p-valor 0,136) y (p-valor 0,00) en estancia hospitalaria, mientras que para la Técnica Endoscópica cervical las desviaciones estándar fueron ( $p$-valor 0,084 y 0,150 respectivamente).

De acuerdo a estos resultados se realizan las pruebas de hipótesis para analizar si la Tiroidectomía Trans-oral está asociada a la reducción tanto de estancia hospitalaria como de tiempos quirúrgicos, aplicando la Distribución T-student en caso de normalidad y la prueba $U$ de MannWhitney de muestras independientes en caso de que no existe normalidad (prueba no paramétrica) Para el Tiempo Quirúrgico promedio se observa que no existe una diferencia significativa entre ambas técnicas con un p-valor de 0,945. Es decir, no existe una reducción del tiempo quirúrgico asociado a la Tiroidectomía Trans-oral.

Para la Estancia Hospitalaria el p-valor ha resultado de 0,461 mayor al nivel de significancia de 0,05 por que se decide aceptar la hipótesis nula, es decir no existen diferencias significativas entre ambas técnicas. Esto es, no existe reducción de los días asociado a la Tiroidectomía Trans-oral.

El Análisis de otras complicaciones según la Técnica empleada se observa que el 52,6\% (N=10/19) no presentó complicaciones para las pacientes con la técnica TOETVA, y el 71,4\% ( $N=10 / 14)$ no presentó otras complicaciones para la Técnica Endoscópica.

\section{Discusión}

La elección de la técnica quirúrgica a emplearse en pacientes con patología tiroidea engloba temas de gran importancia tanto para el paciente, como para la institución de salud encargada de resolver esta patología.

En nuestro país no existe un reporte de enfermedad tiroidea resuelta exclusivamente por cirugía mínimamente invasiva, sin embargo, se tiene conocimiento de estudios comparativos entre cirugía convencional vs mínimamente invasiva, donde concluye que aún se requiere mayor experiencia por parte del cirujano, ya que para la aplicación de las técnicas estudiadas es necesario según Lee et. al que el cirujano realice entre 55 a 60 casos para cualquier variante de cirugía endoscópica, mientras que Fernández et. al concluye que en los 4 primeros años se puede cumplir con los objetivos de la cirugía mínimamente invasiva, sin embargo, para Anuwong ${ }^{(1,4,9)}$ se requiere de una curva de aprendizaje con un estimado de 7 a 10 pacientes en un cirujano con amplia experiencia en tiroidectomía abierta, de esta forma, al evaluar intervenciones quirúrgicas endoscópicas en el país, donde se está iniciando la ejecución de estas técnicas con otros países es notable la diferencia en cuanto a tiempos quirúrgicos, complicaciones y estancia hospitalaria, pero esto forma parte de la curva de aprendizaje del cirujano.

En este estudio se analizan 33 pacientes, los cuales cumplen con los criterios de inclusión, 19 pacientes fueron intervenidos mediante Tiroidectomía Trans-oral laparoscópica TOETVA y 14 pacientes para Tiroidectomía endoscópica; en este caso, la única variante que se realiza en el hospital estudiado es la Tiroidectomía endoscópica cervical (Técnica de Sofferman), la edad promedio varía entre los 28 a 65 años, el $100 \%$ de pacientes estudiados correspondían al sexo femenino, el $42,4 \%$ provenientes de Ibarra y el 15,15\% en Otavalo, estos dos grupos se los ha considerado como los más relevantes, concordando de esta forma con la información proporcionada por el estudio realizado por Pacheco et. al en el año 2005, donde concluye que los niveles más altos de enfermedad tiroidea se encuentran en la zona andina.

En cuanto a las complicaciones presentadas, (14) Anuwong A. tanto en su primer estudio como en los subsecuentes, donde aborda todos los temas relacionados con la técnica TOETVA, concluye que esta tienen buenos resultados postquirúrgicos, disminuyendo la tasa de complicaciones en este período; en el presente estudio se analiza en un grupo complicaciones frecuentes como hipocalcemia, disfonía, hematomas, de esta forma se encuentra que no existe una diferencia significativa en cuanto a complicaciones, la ventaja más relevante entre estas dos técnicas estudiadas, es la ausencia de cicatrices visibles en pacientes intervenidos mediante TOETVA.

La estancia hospitalaria ha sido un tema muy evaluado, en un estudio realizado en el Yale-New Haven Hospital, la estancia hospitalaria para pacientes intervenidos mediante TOETVA fue de 1,1 día, mientras que un estudio comparativo entre TOETVA y Tiroidectomía abierta reporta una estancia hospitalaria de 2 - 3 días, al analizar nuestros datos encontramos que el grupo de pacientes intervenidos por TOETVA tuvo una estancia promedio de 6,32 días, esto debido a una complicación en paciente de 63 años en quien se halla una necrosis de cartílagos traqueales, lo cual se deriva en rotura anterior de los mismos, se coloca bag + traqueostomo y la paciente es trasladada a UCl, donde permanece 
Anuwong et. al 2015

Bangok, Thailand

\section{0 procedimientos}

42 pacientes nódulos tiroides,

22 pacientes - bocios multinodulares

2 enfermedad de graves

2 carcinoma papilar de tiroides La mediana

del tiempo operatorio fue de $115.5 \mathrm{~min}$

(rango 75-300 min)

2 pacientes experimentaron ronquera transitoria, que se resolvió en 2 meses. 1 paciente experimentó un hematoma postoperatorio tardío.

Estancia hospitalaria 3.6 días (rango 2-7 días) después de la operación.

9 pacientes operados entre 2017 - 2018

Todas mujeres 33-64 años

Estancia hospitalaria en el $80 \%$ fue de 1 día.

1 paciente con complicaciones menores en piel.
teSSeroli1; Mauricio

Spagnol2; Álvaro. de Oeste,

Departamento de

Cirugía de Cabeça

e Pescoço,

Chapecó, SC, Brasil

Robert Udelsman, MD,

MBA, FACS, FACE

\section{Deparment of}

Surgery Yale

University School

2016 of Medicine New haven, CT

Yale-New Haven

Hospital New

Haven, CT

\author{
5 pacientes \\ Las indicaciones incluyeron un adenoma \\ tóxico de tiroides, bocios multinodulares, un \\ nódulo tiroideo indeterminado y un \\ microcarcinoma papilar de tiroides \\ Tiempo quirúrgico oscila entre 144 y 296 \\ minutos (media $222 \mathrm{~min}$ ). \\ No hubo casos de lesión recurrente del \\ nervio laríngeo, lesión del nervio mental, \\ hipoparatiroidismo, sangrado, infección, \\ hematoma, seroma, lesión traqueal, \\ enfisema subcutáneo, enfisema \\ mediastínico, epistaxis o conversión a cirugía \\ abierta. \\ Estancia hospitalari 1.1 día \\ No emplearon drenajes y no encontramos \\ seromas o hematomas postoperatorios.
}

por un tiempo aproximado de 24 días, la estancia promedio para la técnica endoscópica cervical fue de 4,14 días.

Mientras que al analizar los tiempos quirúrgicos encontramos que para TOETVA el tiempo promedio fue de 129,84 minutos, con una desviación estándar de $\pm 47,051$, para la técnica endoscópica cervical el tiempo quirúrgico promedio fue de 131,07 minutos con una desviación estándar de $\pm 53,511$, estos resultados relacionados con los tiempos quirúrgicos presentados tanto por el Yale-New Haven Hospital quienes reportan un tiempo quirúrgico de 222 minutos como tiempo promedio, como por Anuwong (4) quien reporta un tiempo quirúrgico de 96,6 minutos, consideramos que las intervenciones analizadas en el presente estudio se encuentran dentro del rango esperado.

\section{Limitaciones}

El presente estudio presenta ciertas limitaciones, inicialmente las relacionadas con estudios retrospectivos, y como segundo punto es que todos los casos fueron del mismo centro hospitalario.

La escasa uniformidad en reportes ecográficos, establecidos por los scores TIRADS o BIRADS, lo cual obstaculizó el análisis estadístico. 


\section{Conclusiones}

- El tiempo quirúrgico entre las dos técnicas estudiadas se encuentra entre los rangos de tiempo descritos en otros estudios, el cual se reduce conforme avanza la curva de aprendizaje.

- En cuanto a las complicaciones presentadas se encontró que los pacientes intervenidos por las dos técnicas estudiadas, más del $52 \%$ no presentaron complicaciones postquirúrgicas, el $48 \%$ de complicaciones no fueron estadísticamente significativas y fueron resueltas a mediano plazo.

- Se concluyó que estéticamente TOETVA no deja cicatrices en el paciente.

\section{Referencias}

1. Anuwong A, Ketwong K, Jitpratoom P, Sasanakietkul T, Duh QY. Safety and Outcomes of the Transoral Endoscopic Thyroidectomy Vestibular Approach. JAMA Surg. 2018 Jan $1 ; 153(1): 21-27$.

2. Yang J, Wang C, Li J, Yang W, Cao G, Wong $\mathrm{HM}$, et al. Complete Endoscopic Thyroidectomy via Oral Vestibular Approach Versus Areola Approach for Treatment of Thyroid Diseases. J LaparoendosC Adv Surg Tech A. 2015 Jun;25(6):470-6.

3. Sivakumar T, Amizhthu RA. Transoral endoscopic total thyroidectomy vestibular approach: A case series and literature review. J Minim Access Surg. 2018 Apr-Jun; 14(2):118-123.

4. Dionigi G, Wu C-W, Tufano RP, Rizzo AG, Anuwong $A$, Sun $H$, Carcoforo $P$, Antonino C. Portinari M, Kim HY. Monitored transoral endoscopic thyroidectomy via long monopolar stimulation probe. Journal of Visualized Surgery. [Internet]. 2018 [citado 23 noviembre 2019]. Disponible en: https://jovs.amegroups. com/article/view/18221/18529

5. Gordillo R, Vasquez W. Tiroidectomía tran soral endoscópica por abordaje vestibula (TOETVA): reporte del primer caso en humanos realizado en Latinoamérica. Rev Chil Cir. 2017, vol.69, n.1, pp.60-64

6. Lavazza M, A. C. Transoral Endoscopic Thyroidectomy Vestibular Approach (TOETVA) with Standardized Neural Monitoring: Tips, Value, and Limits. Scientific Forum: 2017 Clinical Congress [Internet]. 2017 [citado 16 noviembre 2019]. Disponible en: https://www.journalacs.org/article/S1072-7515(17)31237-1/pdf

7. Novelli JL, Novelli F, Maris Batalles S. CIRUGÍA DE TIROIDES, REVISIÓN HISTÓRICA Y NUEVAS TECNOLOGÍAS. Rev. med. Rosario 2017 83(3): 123-127.

8. Tae K, Ji YB, Song CM, Ryu J. Robotic and Endoscopic Thyroid Surgery: Evolution and Advances. Clin Exp Otorhinolaryngol. 2019 Feb;12(1):1-11.

9. Anuwong A. Transoral Endoscopic Thyroidec tomy Vestibular Approach: A Series of the First 60 Human Cases. World J Surg. Marzo de 2016:40, 491-497.

10. Wang, MD, MPH TS, Lyden, MD, MHPE ML, Sosa, MDMA, FACS JA. Thyroidectomy. UpToDate. 1 de mayo de 2019

11. Chai YJ, Kim HY, Kim HK, Jun SH, Dionigi G, Anuwong $A$, et al. Comparative analysis of 2 robotic thyroidectomy procedures: Transoral versus bilateral axillo-breast approach. Head Neck. 2018 May;40(5):886-892.

12. Adam MA, Speicher $P$, Pura J, Dinan MA Reed SD, Roman SA, et al. Robotic thyroidec- tomy for cancer in the US: patterns of use and short-term outcomes. Ann Surg Oncol. 2014 Nov;21 (12):3859-64.

13. Miccoli $P$, Berti $P$, Ambrosini CE. Perspectives and lessons learned after a decade of minimally invasive video-assisted thyroidectomy. ORL J Otorhinolaryngol Relat Spec. 2008;70(5):282-6.

14. Park JO, Park YJ, Kim MR, Sun DI, Kim MS, Koh YW. Gasless transoral endoscopic thyroidectomy vestibular approach (gasless TOETVA). Surg Endosc. 2019 Sep;33(9):3034-3039.

15. Dionigi G, Lavazza $M$, Bacuzzi A, Inversini D, Pappalardo V, Tufano RP, et al. Transoral Endoscopic Thyroidectomy Vestibular Approach (TOETVA): From A to Z. Surg Technol Int. 2017 Feb 7;30:103-112.

6. Tesseroli MAS, Spagnol M, Sanabria Á. Transoral endoscopic thyroidectomy by vestibular approach (TOETVA): initial experience in Brazil. Rev Col Bras Cir. 2018 Nov 14;45(5):e1951.

17. Gallardo-Molina N. The technique and variants of transoral endoscopic thyroidectomy by vestibular approach (TOETVA) to reduce complications. Rev Col Bras Cir. 2019 Mar 7;46(1):e2090.

18. Jitpratoom $P$, Ketwong $K$, Sasanakietkul Anuwong A. Transoral endoscopic thyroidectomy vestibular approach (TOETVA) for Graves' disease: a comparison of surgical results with open thyroidectomy. Gland Surg. 2016 Dec;5(6):546-552.

19. Le QV, Ngo DQ, Ngo QX. Transoral endoscopic thyroidectomy vestibular approach (TOETVA): A case report as new technique in thyroid surgery in Vietnam. Int J Surg Case Rep. 2018;50:60-63.

20. Rosato L, Avenia N, Bernante P, De Palma M, Gulino G, Nasi PG, et al. Complications of thyroid surgery: analysis of a multicentric study on 14,934 patients operated on in Italy over 5 years. World J Surg. 2004 Mar;28(3):2716 .

21. Ross, MD DS. Overview of thyroid nodule formation. UpToDate. 11 de junio de 2019.

22. R Michael Tuttle M. Papillary thyroid cancer. Uptodate. 2019

23. R Michael Tuttle MDSRM. Follicular thyroid cancer. Uptodate. 2019 Mayo 10.

24. Cameselle-Teijeiro JM, Sobrinho-Simões $M$. New WHO classification of thyroid tumors: a pragmatic categorization of thyroid gland neoplasms. Endocrinol Diabetes Nutr. 2018 Mar;65(3):133-135.
25. Vidal O, Saavedra-Perez D, Vilaça J, Pantoja JP, Delgado-Oliver E, Lopez-Boado MA, et al. Minimally-invasive endocrine neck surgery. Cir Esp (Engl Ed). 2019 Jun-Jul;97(6):305-313.

26. Brent, MD GA. Thyroid hormone action. UpToDate. 18 de mayo de 2014.

27. Hwang SH, Kim EK, Moon HJ, Yoon JH, Kwak JY. Risk of Thyroid Cancer in Euthyroid Asymptomatic Patients with Thyroid Nodules with an Emphasis on Family History of Thyroid Cancer. Korean J Radiol. 2016 Mar-Apr;17(2):255-63.

28. Sivakumar T, Amizhthu RA. Transoral endoscopic total thyroidectomy vestibular approach: A case series and literature review. J Minim Access Surg. 2018 Apr-Jun;14(2):1 18-123.

29. Wémeau JL. Nódulo tiroideo. EMC - Tratado de Medicina [Internet]. Enero de 2009 [consultado el 18 de mayo de 20211;13(1):1-5. Disponible en: https://doi.org/10.1016/s16365410(09)70565-3

30. Virella Santana, MD, MS, FCAP, FASCP W. Revista Galenus [Internet]. Nódulos tiroideos con citología indeterminada; [consultado el 18 de diciembre de 2020]. Disponible en: https://www.galenusrevista.com/IMG/ pdf/51-nodulos_tiroideos.pdf

31. BRUNICARDI, BRUNICARDI, BRUNICARDI. Schwartz S Principles Of Surgery Absite And Board Review, 9e. [lugar desconocido]: MCGRAW HILL; 2010.

32. Best $\mathrm{CH}$. Best \& Taylor: Bases fisiológicas de la práctica médica. Buenos Aires: Editorial Médica Panamericana; 2003. 1132 p.

33. Sabiston. Tratado de cirugía : fundamentos biológicos de la práctica quirúrgica moderna - 19. ed. [lugar desconocido]: Elsevier; 2013.

34. Coello C. Edición Médica. El medio de inforamción de salud de Ecuador. [Internet]. Carcinoma de tiroides el más común entre las mujeres ecuatorianas: 3 de octubre de 2017 mujeres ecuatorianas; 3 de octubre de 2017 ponible en: https://www.edicionmedica.ec/ secciones/salud-publica/carcinoma-de-tiroides-el-m-s-com-n-entre-las-mujeres-ecuatorianas-91042

35. Horvath DE Contacto Científico [Internet]. Vista de Clasificación TIRADS - una herramienta útil en la selección de nódulos tiroídeos que requieren punción diagnóstica; 2012 [consultado el 18 de diciembre de 2020]. Disponible en: https://contactocientifico.alemana.cl/ojs/index.php/cc/article/ view/81/83 